\title{
The psyche and the soma - redefining the role of the psychiatrist in medical care
}

The theme of the 15th biannual national congress of the South African Society of Psychiatrists (SASOP) is 'The Psyche and the Soma'. This was chosen to reflect the growing awareness among medical practitioners of psychiatric disorders as multi-systemic disorders, rather than diseases of the central nervous system (CNS) only. It is also hoped that it will be a signpost on the road to the reintegration of psychiatry into general medicine in South Africa, as is happening in the rest of the world. The 're-launch' of psychiatry as part and parcel of general medical services is arguably long overdue. Our speciality has over many decades become 'divorced' from the rest of the health care services, and it is now recognised that this has been to the detriment of both psychiatry and the rest of medicine.

Although the trend has been for medicine to become more and more specialised, many have been asking whether this siloapproach is in fact serving the best interests of the public. By compartmentalising medical disorders we may run the risk of losing our ability to recognise and manage conditions that may be causing, result from or exist as co-morbid conditions with the disorders that we are managing

As a consequence of this new view of psychiatric illness, we have for example come to realise that major depressive disorder (MDD) is a systemic disorder with wide-ranging effects on systems outside of the CNS. Through among others its effects on the hypothalamic-pituitary-adrenal axis, MDD may play a role in loss of normal heart rate variability, and may increase the risk of thrombosis, insulin resistance, hypertension and atherosclerosis. The INTERHEART study has in fact shown psychosocial factors (including depression) to be important, independent risk factors for heart disease.'

Although there is growing recognition of the interaction of psychiatric disorders and other medical disorders, clinicians who work outside the field of mental health are reluctant to manage patients who have mental illness. Psychiatrists therefore tend to become - albeit reluctantly at times - responsible for managing both the psychiatric and general medical care of patients with severe mental illness. There is considerable evidence that patients who suffer from mental illness face many obstacles to the diagnosis and treatment of co-morbid medical disorders. Patients with mental illness and co-morbid medical disorders find it very difficult to access medical services, and even when they are able to gain access to these services they receive poorer quality of care than patients without mental illness. ${ }^{2}$

This discrimination is sharply reflected in the morbidity and mortality statistics: Patients with mental illness die approximately
20 years earlier than their counterparts in the general population. Although suicide plays some role, the vast majority of patients with severe mental illness die of natural causes. In fact, the principal cause of death in patients with severe mental illness is cardiovascular disease. Although cardiovascular disease is set to become the major cause of mortality across the world, it is even more prevalent in patients with severe mental illness. Patients who suffer from depression, bipolar disorder or schizophrenia and who also have a medical disorder have much higher standard mortality ratios than subjects with medical disease alone. ${ }^{3}$

The fact that cardiovascular disease and metabolic disorders are so prevalent in patients with severe mental illness is of great importance to psychiatrists. Not only should we equip ourselves to be able to search for, identify and manage these conditions, but we should also keep them in mind when prescribing treatment. It is a well-publicised fact that patients in large treatment trials have very high prevalence rates of cardiometabolic disorder. In the now infamous CATIE study, $43 \%$ of patients met criteria for metabolic syndrome at baseline. ${ }^{4}$ The contribution that psychotropic medications make to these disorders has therefore also become a major focus of attention. It is no longer acceptable for psychiatrists only to consider the patient's mental state when deciding on pharmacological treatment; we also have to consider the long-erm effects that the medication will have on the rest of the body. Although a lot of attention has been focused on the effects of the second-generation antipsychotics, there is also evidence emerging for the effects (both positive and negative) of other psychotropic drugs, such as antidepressants, on cardiometabolic health in particular.

During much of the 20th century psychiatry moved away from the rest of medicine, preferring to use different treatments, approaches and settings. From the 1980s onward this process has slowly been reversed, although there is still some way to go and considerable resistance remains on both sides. The current research evidence shows that we have no alternative: mental disorders are so closely interwoven with other medical conditions that they cannot be treated as completely separate entities. The reciprocal effects of disease and treatment should be considered by both psychiatrists and other health practitioners. Although it cannot be expected that psychiatrists should have in-depth knowledge of all medical conditions, we should at least have a working knowledge of the conditions that are commonly associated with psychiatric disorders. These include all the components of the metabolic syndrome, thyroid disease and cardiovascular disease. The role of the psychiatrist in the 
management of the physical disorders that accompany psychiatric illness is to recognise, intervene in the acute phase if necessary, refer appropriately, interact with colleagues, and manage chronic care and medication interactions. It is an exciting challenge that will not only re-acquaint us with the roots of our training but also improve the lives of those we serve immeasurably.

\section{Piet Oosthuizen}

Dana Niehaus

Department of Psychiatry

Stellenbosch University

Tygerberg, W Cape

\section{References}

1. Yusuf S, Hawken S, Ounpuu S, et al. Effect of potentially modifiable risk factors associated with myocardial infarction in 52 countries (the INTERHEART study): casecontrol study. Lancet 2004; 364: 937-952

2. Frayne $S M$, Halanych JH, Miller DR, et al. Disparities in diabetes care - impact of mental illness. Arch Intern Med 2005: 165: 2631-2638.

3. Newcomer JW. Medical risk in patients with bipolar disorder and schizophrenia. J Clin Psychiatry 2006; 67: 25-30

4. McEvoy JP, Meyer JM, Goff DC, et al. Prevalence of the metabolic syndrome in patients with schizophrenia: Baseline results from the Clinical Antipsychotic Trials of Intervention Effectiveness (CATIE) schizophrenia trial and comparison with nationa estimates from NHANES III. Schizophr Res 2005; 80: 19-32

\section{Clinical Trials in Psychopharmacology Pre-Congress Workshop}

\section{SASOP 2008 Congress Sunday August 10, 14h00-17h00}

This workshop will focus on general problems and challenges of designing and implementing clinical trials. Topics to be covered include trial design, diagnosis, clinical assessment, patient ascertainment, and recruitment. The workshop will also review recent developments in psychotropic drug research and ethical and regulatory issues in the conduct and reporting of clinical trials. It is intended for psychiatrists, registrars and other interested persons in academic and private settings who are involved in psychopharmacology clinical trials or who would like to become involved.

\section{Provisional Program}

14h00-14h15: Design, Conduct and Implementation of Clinical Trials Soraya Seedat, University of Stellenbosch

14h15-14h45: Diagnostic Assessment, Rating Scales and Methodological Issues Catherine Maud, Durban. Private Practice

14h45-15h00: Patient Selection in Clinical Trials: Critical to Successful Outcomes Andre Joubert, Lundbeck Institute, Denmark

15h00-15h20: Placebo Response in Clinical Trials: What Can We Do About It? Robin Emsley, University of Stellenbosch

\section{Tea: 15h20-15h30}

15h30-15h50: Ethical and Regulatory Issues: Recent Developments, Informed Consent, Patient Ascertainment and Recruitment Felix Potocnik, University of Stellenbosch

15h50-16h10: Ethics of Placebo-Controlled Studies in Developing Countries to Fulfill EU Regulations Andre Joubert, Lundbeck Institute, Denmark

16h10-16h30: Engaging Industry: Contracting and Negotiating Clinical Trials Frans Korb, Eli-Lilly, South Africa

16h30-17h00: Quiz and Q \& A 